\title{
Usefulness of 16-Slice Multislice Spiral Computed Tomography for Follow-up Study of Coronary Stent Implantation
}

\author{
Makoto Watanabe, MD; Shiro Uemura, MD; Hajime Iwama, MD; Satoshi Okayama, MD; \\ Yukiji Takeda, MD; Hiroyuki Kawata, MD; Manabu Horii, MD; Tamio Nakajima, MD; \\ Shinji Hirohashi, MD*; Kimihiko Kichikawa, MD*; \\ Akira Ookura, MD**; Yoshihiko Saito, MD
}

\begin{abstract}
Background Although multislice spiral computed tomography (MSCT) is a promising technique for noninvasive coronary angiography, its usefulness in patients with stent implantation remains unclear. The aim of the present study was to compare the usefulness of MSCT with that of invasive coronary angiography for evaluating coronary stent patency.

Methods and Results Thirty-one patients were enrolled after coronary stent implantation. Sixteen-slice MSCT scans were performed (39.0 \pm 21.8 days) before follow-up coronary angiography. After assigning an image score based on luminal visibility ( $1=$ poor, $2=$ fair, $3=$ good), factors determing image quality were analyzed. Among 42 implanted stents, $33(78 \%)$ were assigned an image score of 3, $2(5 \%)$ a score of 2, and $7(17 \%)$ a score of 1. Image scores among stents with diameters $\geq 3.5 \mathrm{~mm}$ were significantly $(\mathrm{p}<0.05)$ higher than among smaller stents $(\leq 3.0 \mathrm{~mm})$. Stent strut thickness did not affect image quality, but coronary calcification significantly $(\mathrm{p}<0.01)$ hampered the image quality. After excluding 7 stents with image scores of 1 , the sensitivity, specificity, positive and negative predictive values of MSCT to identify patent stents were $83 \%, 90 \%, 63 \%$ and $96 \%$, respectively.

Conclusions MSCT can provide useful and valuable clinical information for assessing stent patency during the follow-up period when patients are treated with relatively large diameter coronary stents. (Circ J 2006; 70: 691-697)
\end{abstract}

Key Words: Coronary artery disease; Coronary stent; Multislice spiral computed tomography

$\mathbf{I}$ $\mathrm{n}$ recent years, coronary stent implantation has become an established method for treating patients with coronary artery disease. However, in-stent restenosis attributable to intimal hyperplasia still occurs at a relatively high rate, not only with bare metal stents but also with drug eluting stents 1,2 Conventional coronary angiography is still the domain of the assessment of 'in-stent' restenosis. However, angiography has limitations because of its invasiveness and high medical expense. Thus, a non-invasive alternative to assess the patency of the stented arterial segment would be highly desirable.

The application of multislice spiral computed tomography (MSCT) for imaging the coronary vasculature has been continuously evolving. With increasing numbers of detector rows (currently as many as 64), computed tomography (CT) scanners now provide coronary arterial images with markedly improved temporal and spatial resolution. In vitro and phantom studies on stent imaging have shown that 16-slice MSCT can adequately resolve even the lumens of stents placed within small vessels? In clinical settings, however, many factors, including motion artifacts because of arrhythmia or high heart rate, coronary calcification, and

(Received November 4, 2005; revised manuscript received February 27, 2006; accepted March 17, 2006)

First Department of Medicine, *Department of Radiology, Nara Medical University, Kashihara and **Department of Radiology, Kokuho Central Hospital, Shiki, Japan

Mailing address: Shiro Uemura, MD, First Department of Medicine,

Nara Medical University, 840 Shijo-cho, Kashihara 634-0813, Japan artifacts as a result of metallic material, can impair the quality of images of implanted stents. The purpose of the present study, therefore, was to determine: (i) the usefulness of 16-slice MSCT for evaluating coronary stent patency in a clinical population; and (ii) the parameters that determine the quality of stent images.

\section{Methods}

Patients and Study Protocol

The study consisted of 31 patients (27 male, 4 female; average age $64 \pm 10$ years; 26 patients with old myocardial infarction, 5 with stable angina) who had been treated with coronary artery stent implantation (42 total stents) between November 2002 and August 2004. The patients were scheduled for follow-up invasive coronary angiography between June 2003 and November 2004. Before their angiography, each patient underwent MSCT to assess coronary stent patency. All patients were in sinus rhythm at the time of the CT angiography, and there were no contraindications to CT imaging. Twenty-five patients had a prescan heart rate of $>70$ beats/min; they were given a single oral dose of 20-40 mg metoprolol 90-120 min before the CT examination. This protocol was approved by our Institutional Ethics Committee (\#2002-009, Nara Medical University Ethics Committee), and written informed consent was obtained from all patients. 


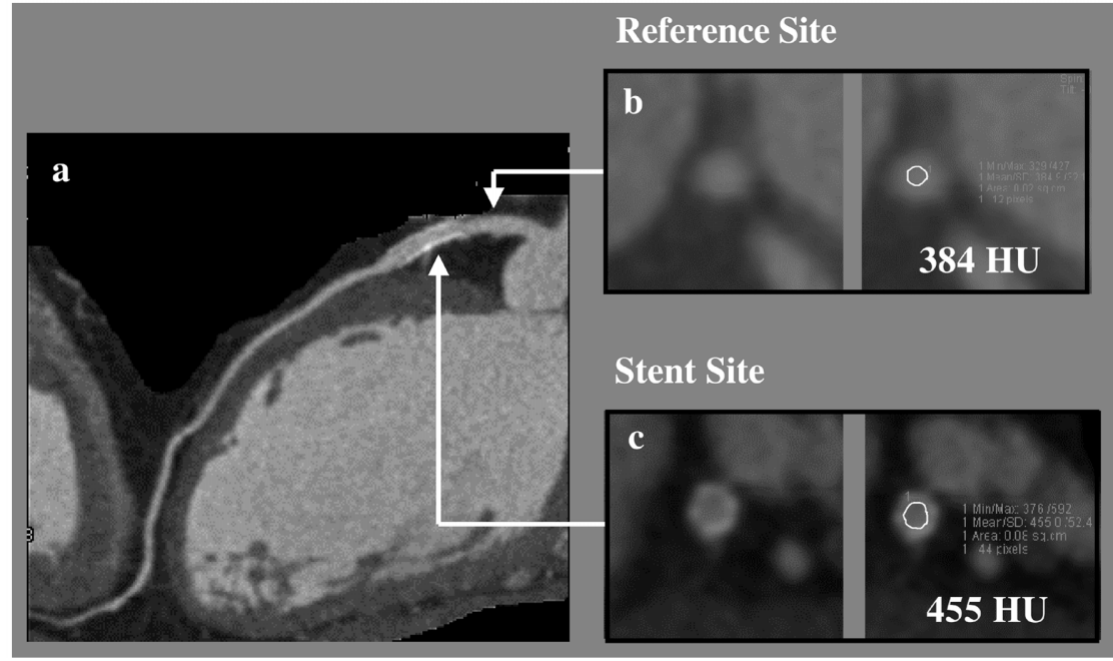

Fig 1. (a) Curved multiplanar reconstruction (MPR) image shows a implanted stent in left anterior decending artery. (b) MPR image shows the cross-sections of proximal site outside stent (reference site). Computed tomography (CT) attenuation value was calculated 384 Hounsfield units (HU) in reference site. (c) MPR image shows the crosssections of in-stent lumen (stent site). CT attenuation value was calculated $455 \mathrm{HU}$ in in-stent site.

\section{MSCT Scan Protocol}

Coronary CT angiography was carried out using a 16slice MSCT scanner (Sensation 16, Siemens, Forchheim, Germany) with a collimation of $16 \times 0.75 \mathrm{~mm}$ and $0.42 \mathrm{~s}$ rotation time. The tube current was $250 \mathrm{~mA}$ at $120 \mathrm{kV}$. For contrast enhancement, $75 \mathrm{ml}$ of contrast media (Iopamiron $370 \mathrm{mg} / \mathrm{ml}$ ) were administered intravenously at rate of $3 \mathrm{ml} / \mathrm{s}$, followed by a $30 \mathrm{ml}$ saline chaser bolus administered using a power injector. Images were then acquired during an inspiratory breath hold. An electrocardiogram (ECG) was simultaneously recorded during the MSCT examination for retrospective gating of data. ECG-gated image reconstruction was carried out using a medium-sharp body convolution kernel (B30f) in the mid- to end-diastolic phase of the cardiac cycle using between $30 \%$ and $70 \%$ relative delay.

\section{Quantitative Invasive Coronary Angiography}

Conventional coronary angiograms were evaluated by an experienced observer without knowledge of the data gathered using MSCT. Quantitative coronary angiography (QCA) served as the gold standard for stenosis detection. Adequate stent patency on QCA was defined as in-stent or peri-stent stenosis reducing luminal diameter by less than $50 \%$.

\section{Data Analysis}

To assess the patency of coronary stents, images produced by multiplanar reconstruction (MPR) and/or curved MPR were evaluated by 2 experienced observers (reader 1 and 2) unaware of the catheterization results. Initially, each stent was assigned an image score of 1 (poor image quality or uninterpretable), 2 (adequate image quality), or 3 (good image quality) based on the classification mentioned by Schuijf et al. Stent images with a score of 2 or 3 were evaluated and included in our statistical analysis, but those with a score of 1 were not. Once an image score was assigned and stent patency evaluated, we analyzed factors determining the quality of the MPR images, including stent diameter, stent strut thickness and coronary calcification. To assess the effects of specific characteristics on image quality, stents were categorized by diameter $(2.5 \mathrm{~mm}$, $3.0 \mathrm{~mm}, 3.5 \mathrm{~mm}$, or $4.0 \mathrm{~mm}$ ) and as having either thick struts ( $\geq 0.005$ inches; Trister stent, BX velocity stent, S670 stent) or thin struts $(<0.005$ inches; Penta stent, Duraflex stent).

Implanted stents were deemed adequately patent when the longitudinal MPR images revealed: (i) no disruption of coronary flow distal to the implanted stent; (ii) peri-stent stenosis was no more than $50 \%$; and (iii) there was no contrast defect in the stent. Stents not meeting at least one of the 3 criteria were regarded as restenosis. In the case (image score 3 ) that had difficulty with evaluation of restenosis in the stent by longitudinal MPR re-construction, cross-sectional MPR images of the target stents were also examined. In cross-section analysis, we calculated the area stenosis ([vessel area: inner margin of stent artifact] - [contrast enhanced area]/vessel area $\times 100$ ). More than $50 \%$ area stenosis was also regarded as restenosis. Inter-observer agreement in the interpretation of the stent restenosis was high ( $\mathrm{K}=0.77)$ among 35 stented segnents, and higher $(\mathrm{K}=$ 0.86 ) among 21 stents $\geq 3.5 \mathrm{~mm}$ in diameter (Cohen $\mathrm{K}$ statistics).

Furthermore, to quantitatively evaluate the luminal patency in the coronary stent, the CT attenuation values of the minimum luminal site within the stent lumen (stent site) and the normal luminal site outside the stent (reference site) were measured with a region-of-interest (ROI) technique in the cross-sections of target vessels. The ROI of the stent site was placed by using the free-hand trace in stent lumen without inclusion of the stent struts and the ROI of the reference site was placed in the center of normal luminal site (the size of all ROI with 12 pixels, Fig 1). The relative value of the stent site was expressed as the equation (stent site attenuation values/reference site attenuation value). Then CT attenuation measurements were compared between restenotic stent lumens and non-restenotic stent lumens.

\section{Statistical Analysis}

Data are presented as means \pm standard deviation and were compared by analysis of variance followed by Fisher's protected least significance difference test. Average image scores were then calculated for each category. Sensitivity, specificity, positive and negative predictive value for detection of stent patency (also determined by QCA) were determined for each stent. Multivariate logistic regression analysis was performed to determine independent factors determining the quality of images of stent lumens. Values of $\mathrm{p}<0.05$ were considered significant. 


\section{Results}

\section{Clinical Characteristics of the Study Group}

The clinical characteristics of the patients and the coronary stents used are summarized in Table 1 . The average interval between MSCT and conventional angiography was $39.0 \pm 21.8$ days. At the time of MSCT, the mean heart rate was $54 \pm 6$ beats $/ \mathrm{min}$ (range: 40 to 71 beats $/ \mathrm{min}$ ). The mean scan duration was $19 \pm 2 \mathrm{~s}$ (range: 14 to $25 \mathrm{~s}$ ). MSCT was performed $6.2 \pm 4.0$ months (range: 3 to 24 months) after stent implantation in 28 patients (38 stents) and $8.3 \pm 3.2$ months (range: 5 to 11 months) after target vessel revascularization for in-stent restenosis in 4 patients (4 stents). MSCT was performed successfully in all subjects. Sixteen stents were implanted in the left anterior descending coronary artery, 9 in the left circumflex coronary artery and 17 in the right coronary artery. The diameters of the implanted stents ranged from 2.5 to $4.0 \mathrm{~mm}$ : 5 stents were $2.5 \mathrm{~mm}$ in diameter; 5 stents were $3.0 \mathrm{~mm} ; 16$ stents were $3.5 \mathrm{~mm}$; and 9 stents were $4.0 \mathrm{~mm}$. Five different types of stent had been used: 28 Multilink Penta (Guidant), 4 Multilink Trister (Guidant), 3 Bx-Velocity (Cordis), 3 S670 (Medtronic) and 4 Duraflex (Goodman).

\section{Visibility of Coronary Stent Lumens}

Based on the image quality, 7 stents (17\%) were assigned an image score of $1,2(5 \%)$ an image score of 2 , and 33 (78\%) an image score of 3 . Fig 2 shows representative cases for each image score. We next assessed the factors that could potentially affect image quality. Examination of the relationship between stent diameter and the quality of the stent images showed that as stent diameter declined, there was a significant decline in image quality (Figs 3,4; Table 2). Similarly, calcification at the stent site significantly $(p<0.01)$ diminished the quality of luminal images of implanted stents (Table 2). Coronary artery calcification at the stent site was detected in 12 stents. The average image score was $2.4 \pm 1.0$ for stents with calcification and $2.7 \pm 0.7$ for stents without calcification. However, strut thickness had no significant effect on image quality (Fig 5, Table 2).

\section{Evaluation of Stent Patency}

Before evaluating the luminal patency of implanted coronary stents during the chronic phase, 7 stents receiving an image score of 1 were disallowed because of poor image quality. The causes of poor quality were small diameter $(2.5 \mathrm{~mm})$ in 4 cases, severe calcification around the stent
Table 1 Clinical Characteristics of the Study Population $(n=42)$

\begin{tabular}{lc}
\hline \hline$M / F$ & $27 / 4$ \\
Age (years) & $64 \pm 10$ \\
Heart rate (beats/min) & $54 \pm 6$ \\
Scan duration $(s)$ & $19 \pm 2$ \\
Stent location & \\
LAD & $16(38 \%)$ \\
LCX & $9(22 \%)$ \\
RCA & $17(40 \%)$ \\
Stent diameter $(\mathrm{mm})$ & \\
2.5 & $5(12 \%)$ \\
3.0 & $16(38 \%)$ \\
3.5 & $12(29 \%)$ \\
4.0 & $9(21 \%)$ \\
Stent type & \\
Multilink Penta (Guidant) & $28(68 \%)$ \\
Multilink Trister (Guidant) & $4(9 \%)$ \\
Bx-velocity (Cordis) & $3(7 \%)$ \\
S670 (Medtronic) & $3(7 \%)$ \\
Duraflex (Goodman) & $4(9 \%)$ \\
\hline
\end{tabular}

$L A D$, left anterior descending coronary artery; LCX, left circumflex coronary artery; $R C A$, right coronary artery.

in 2 cases, and a motion artifact in 1 case. Among the 35 stents receiving an image score of 2 or 3, 27 were deemed to be adequately patent based on MSCT examination; the other 8 were diagnosed as having an in-stent or peri-stent restenotic lesion (Fig 6). In the QCA study, by contrast, 6 of the 35 stents were diagnosed as having in-stent or peristent restenosis. One in-stent restenotic lesion was underestimated using MSCT, despite adequate image quality, and restenosis was overestimated in 3 stents using MSCT and were counted as false-positives ( 2 of those stents had calcifications). Among the 35 stents receiving an image score 2 or 3, the sensitivity of MSCT for evaluating stent patency during the chronic phase was $83 \%$ (reader 1) and $100 \%$ (reader 2), specificity was 90\% (reader 1) and 93\% (reader 2 ), positive predictive value was $63 \%$ (reader 1 ) and $75 \%$ (reader 2), and negative predictive value was $96 \%$ (reader 1) and $100 \%$ (reader 2).

We also separately assessed the diagnostic accuracy of MSCT in patients with larger stents $(\geq 3.5 \mathrm{~mm})$ because all stents $\geq 3.5 \mathrm{~mm}$ in diameter showed good image quality (score 3), and because the in-stent lumen of some smaller stents $(\leq 3.0 \mathrm{~mm})$ tended to be obscured by stent-related artifacts or coronary calcification. Among 21 stents $\geq 3.5 \mathrm{~mm}$ in diameter, the sensitivity was $100 \%$ (reader 1 ) and $100 \%$
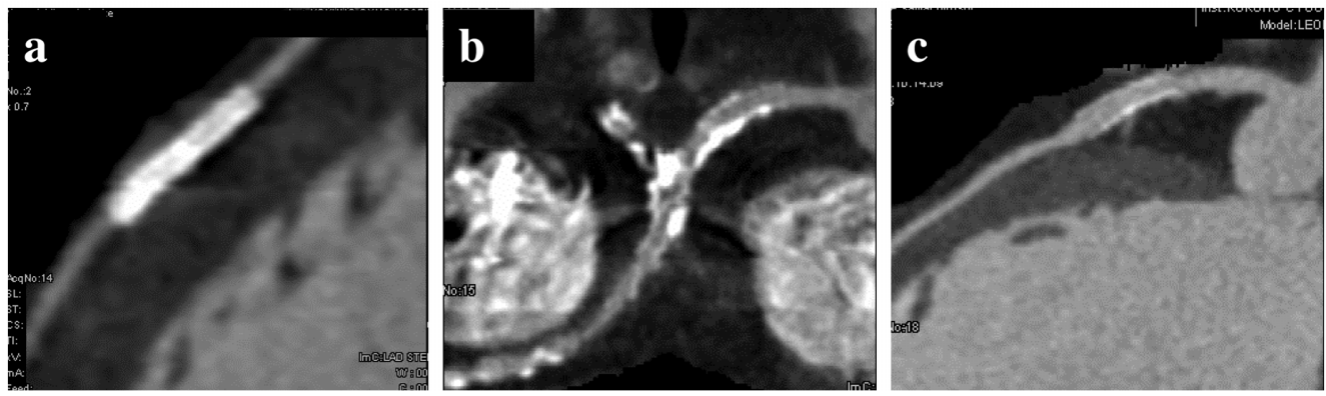

Fig 2. Curved multiplanar reconstruction images showing representative cases based on image score. (a) Image score 1 (poor image quality or uninterpretable); $2.5 \mathrm{~mm}$ diameter stented segment (Multilink Penta, Guidant). (b) Image score 2 (adequate image quality); $3.0 \mathrm{~mm}$ and $3.5 \mathrm{~mm}$ diameter stented segments (Multilink Penta, Guidant) with severe calcification. (c) Image score 3 (good image quality); $4.0 \mathrm{~mm}$ diameter stented segment (Multilink Penta, Guidant). Conventional angiography revealed that (a), (b), and (c) did not exhibit in-stent restenosis. 

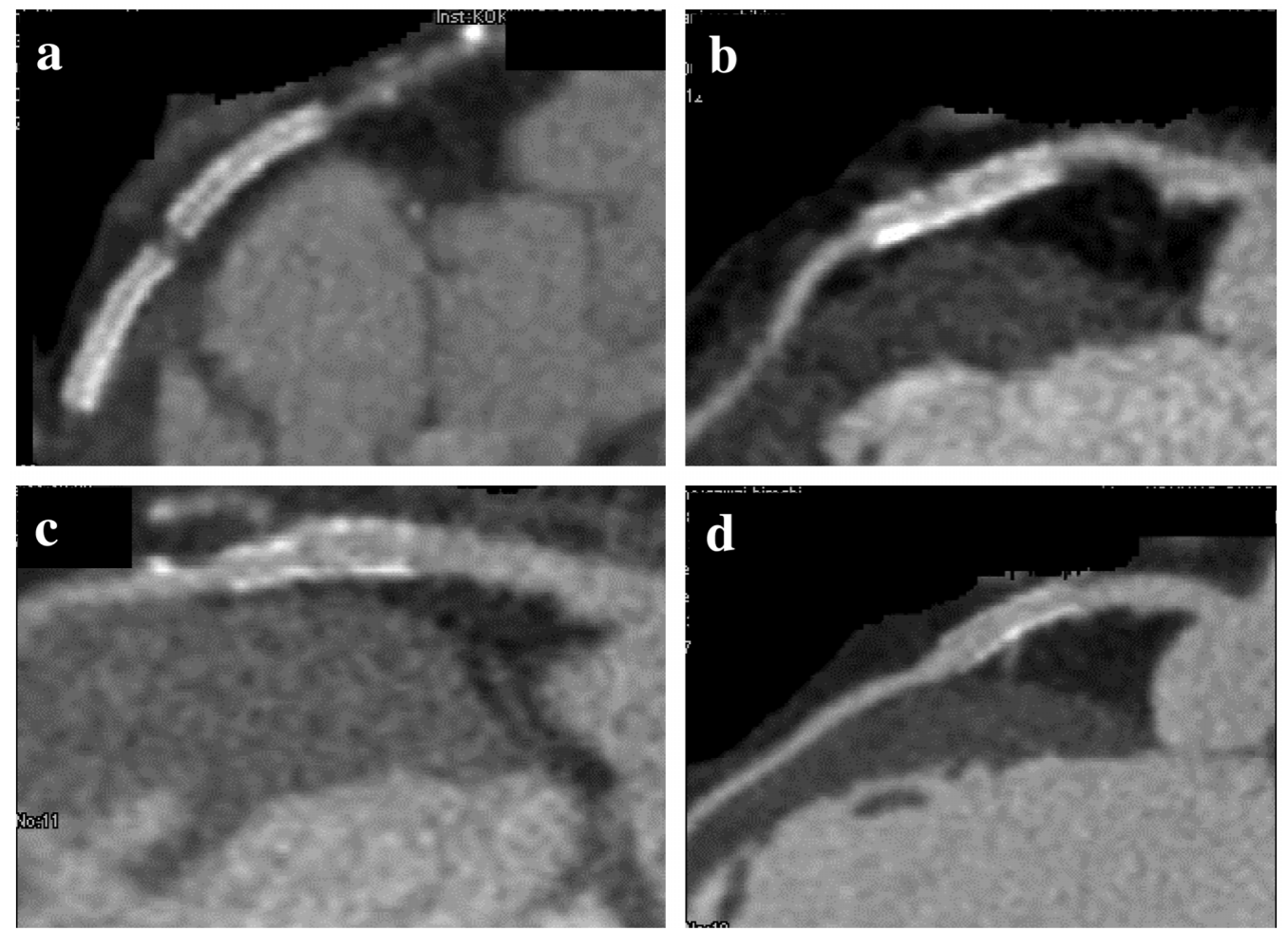

Fig 3. Curved multiplanar reconstruction images of stents of differing diameters. (a) $2.5 \mathrm{~mm}$ diameter stent (Multilink Penta, Guidant) in the left circumflex coronary artery, assigning it image score 1. (b) $3.0 \mathrm{~mm}$ diameter stent (Multilink Penta, Guidant) in the proximal left anterior descending coronary artery (LAD), assigning it image score 3. (c) $3.5 \mathrm{~mm}$ diameter stent (Multilink Penta, Guidant) in the proximal LAD, assigning it image score 3. (d) $4.0 \mathrm{~mm}$ diameter stent (Multilink Penta, Guidant) in proximal the LAD, assigning it image score 3. Conventional angiography revealed that (a), (b), (c), and (d) did not exhibit in-stent restenosis.

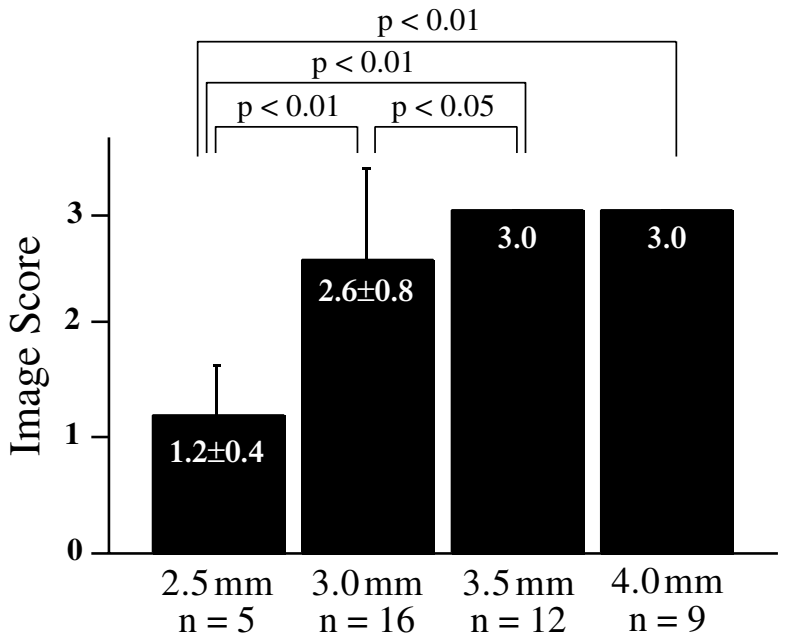

Fig 4. Effect of stent diameter and image score. Image scores obtained with larger stents $(3.5 \mathrm{~mm}$ and $4.0 \mathrm{~mm})$ were significantly higher than those obtained with smaller stents $(2.5 \mathrm{~mm}$ and $3.0 \mathrm{~mm})$.

(reader 2), specificity was $89 \%$ (reader 1) and 94\% (reader 2 ), positive predictive value was $60 \%$ (reader 1) and $75 \%$ (reader 2), and negative predictive value was $100 \%$ (reader 1) and $100 \%$ (reader 2) (Table 3$)$.

\section{CT Attenuation Values of In-Stent Lumen}

With conventional coronary angiography, 6 stents were diagnosed as restenosis and 29 stents as non-restenosis.
Table 2 Factors Influencing the Lumen Visibility of Coronary Artery Stent by Multivariate Logistic Regression Analysis

\begin{tabular}{lccc}
\hline \hline & $\begin{array}{c}\text { Image } \\
\text { score } 1 \\
n=7\end{array}$ & $\begin{array}{c}\text { Image } \\
\text { score 2,3 } \\
n=35\end{array}$ & p value \\
\hline $\begin{array}{l}\text { Stent diameter } \\
(\text { mm })\end{array}$ & $2.7 \pm 0.3$ & $3.4 \pm 0.4$ & $<0.01$ \\
$\begin{array}{l}\text { Stent strut thickness } \\
\text { (inch) }\end{array}$ & $0.0043 \pm 0.0009$ & $0.0040 \pm 0.0006$ & $N S$ \\
$\begin{array}{l}\text { Coronary artery } \\
\text { calcification }\end{array}$ & $3(43 \%)$ & $9(26 \%)$ & $<0.01$ \\
\hline
\end{tabular}

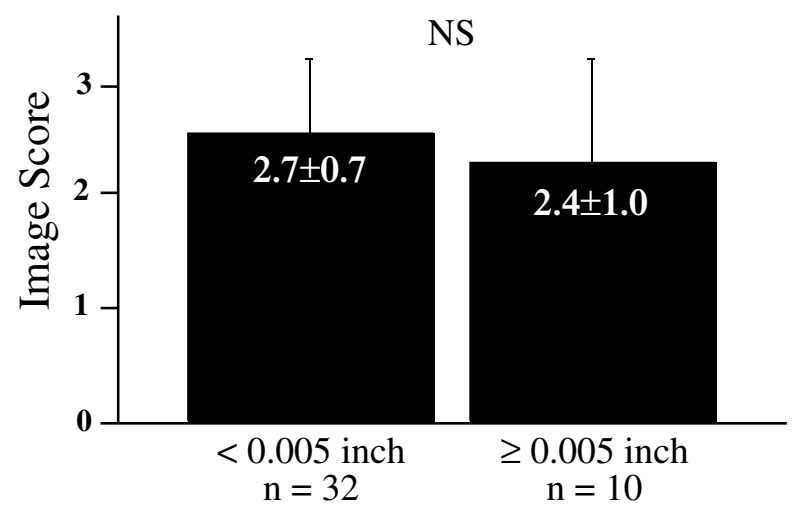

Fig 5. Effect of stent strut thickness and image score. Stent strut thickness had no significant effect on image score. 

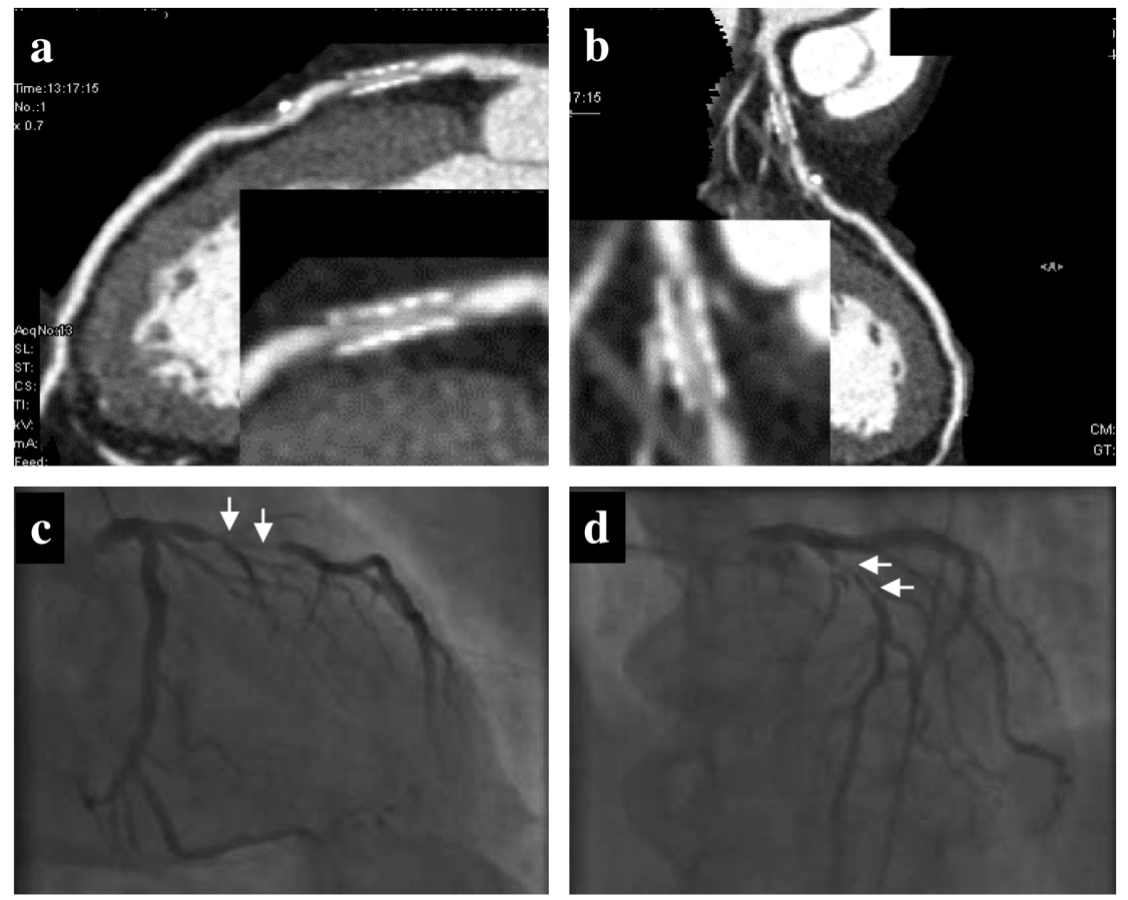

Fig 6. Representative case of in-stent restenosis. A $3.5-\mathrm{mm}$ diameter stent (Multilink Penta, Guidant) had been implanted in the proximal left anterior descending coronary artery 5 months earlier. Curved multiplanar reconstruction show severe narrowing in the stent lumen $(\mathrm{a}, \mathrm{b})$. Conventional coronary angiography also showed significant in-stent restenosis (arrow) (c, d).

Table 3 Diagnostic Accuracy to Detect the Coronary Artery Stents Patency

\begin{tabular}{lccc}
\hline \hline & $\begin{array}{c}\text { Image score 2,3 } \\
n=35\end{array}$ & & $\begin{array}{c}23.5 \mathrm{~mm} \text { stent } \\
n=21\end{array}$ \\
\cline { 2 - 2 } Sensitivity & Reader 1/Reader 2 & & Reader 1/Reader 2 \\
Specificity & $83 / 100 \%$ & & $100 / 100 \%$ \\
Positive predictive value & $90 / 93$ & & $89 / 94$ \\
Negative predictive value & $63 / 75$ & & $60 / 75$ \\
$\mathrm{~K}$ value & $96 / 100$ & & $100 / 100$ \\
& 0.77 & & 0.86 \\
\hline
\end{tabular}

The mean attenuation value of stent site measured on the restenotic stents $(227.5 \pm 70.3)$ was significantly lower than that on the non-restenotic stents $(409.9 \pm 84.1 ; \mathrm{p}<0.01)$. Similarly, the mean relative value on the restenotic stents $(0.68 \pm 0.23)$ was significantly lower than that on the nonrestenotic stents $(1.24 \pm 0.19 ; \mathrm{p}<0.01)$. In particular, all 5 patients whose relative value was less than 0.9 were shown to have in-stent restenosis. These results indicate that larger amount of contrast agent was filled in non-restenotic stents compared to restenotic stents (Table4).

\section{Discussion}

Over the past 15 years, coronary artery stenting has become an established medical treatment for patients with ischemic heart disease, and its use continues to increase rapidly, as the incidence of long-term restenosis is significantly lower with stenting than with ordinary balloon angioplasty5 Nonetheless, the in-stent restenosis rate is still high (20-30\%) for bare metal stents, 5 ,6 wich usually necessitates follow-up examination using coronary angiography. Although conventional coronary angiography is still considered as the "Holy Grail" for the diagnosis of coronary artery disease? recent development in diagnostic modalities such as magnetic resonance angiography (MRA), electron beam CT (EBCT), and MSCT has allowed non-invasive
Table 4 Comparison of CT Attenuation Measurements With Restenotic and Non-Restenosed Stent Lumen

\begin{tabular}{|c|c|c|c|}
\hline & $\begin{array}{c}\text { Restenotic } \\
\text { stents } \\
n=6\end{array}$ & $\begin{array}{c}\text { Non-restenotic } \\
\text { stents } \\
n=29\end{array}$ & $p$ values \\
\hline Reference Site (HU) & $345.5 \pm 60.7$ & $333.4 \pm 55.8$ & NS \\
\hline Stent Site $(H U)$ & $227.5 \pm 70.3$ & $409.9 \pm 84.1$ & $<0.01$ \\
\hline $\begin{array}{l}\text { Relative values } \\
\text { (Stent Site/Reference Site) }\end{array}$ & $0.68 \pm 0.26$ & $1.24 \pm 0.19$ & $<0.01$ \\
\hline
\end{tabular}

CT, computed tomography; HU, Hounsfield units.

visualization of the coronary artery. MRA might be the most promising method because it does not require radiation or contrast media, but its spatial resolution is still limited8 Moreover, visualization of coronary artery stents is not possible by MRA? EBCT also has limitations in spatial resolution and it is not suitable for the assessment of stent patency?

With the development of MSCT, however, it has become possible to visualize the precise anatomy of the moving heart ${ }^{10-12}$ Moreover, the recently introduced 16-slice MSCT system, which allows simultaneous acquisition of 16 submillimeter slices, enables more detailed imaging, not only of stenosed coronary arteries but also of stents implanted within coronary arteries! $!^{3}$ Our aim, therefore, was to evaluate the usefulness of 16-slice MSCT for clinical evaluation of the patency of metallic stents within coronary arteries. Our findings using 16-slice MSCT indicate that comparatively small stent diameters $(\leq 3.0 \mathrm{~mm})$ and coronary calcification, but not stent strut thickness, significantly impair imaging of the stent lumen using 16-slice MSCT. It has been previously reported that in unmoving organs, such as brain or liver, arteries with diameters as small as $1.0 \mathrm{~mm}$ can be imaged using 16-slice MSCT ${ }^{14}$ In the case of coronary arteries within a moving heart, there is sufficient temporal and spatial resolution to image coronary stents with diameters $\geq 3.5 \mathrm{~mm}$. 
It was previously reported that with 4-slice MSCT even minor calcification can significantly impair the quality of coronary artery images ${ }^{10,15,16}$ By contrast, more reports have shown that the quality of coronary artery images is not significantly impaired by minor calcification when using 16-slice MSCT!7 In our study population, multiple regression analysis revealed that coronary arterial calcification at the stent site significantly reduced the quality of luminal images; however, images of only 1 of 12 stents with calcification was determined to be uninterpretable as a result of the severity of the calcification. However, when a stent is implanted within a coronary segment with calcification, especially with heavy calcification, it is likely that 16-slice MSCT will not be sufficient to obtain interpretable images of the stent lumen.

The results of our multivariate logistic regression analysis indicate that stent strut thickness does not affect image quality. By contrast, Schuijf et al suggested that thicker struts tended to make images uninterpretable because stents with thicker struts were more affected by partial volume effect because of high-density texture. The reason for the discrepancy between our findings and those of Schuijf et al is not clear, although several factors related to the scanning conditions could influence the severity of the artifact from metallic stents. It is noteworthy, for instance, that heart rate at the time of CT examination was substantially lower in our study population than in theirs $(54 \pm 6$ vs $65 \pm 11$ beats $/ \mathrm{min})$.

In stents with good image quality, we found that the sensitivity and specificity of 16-slice MSCT were sufficient to assess coronary artery stent patency. In particular, the presence of contrast enhancement of the vessel distal to the stent was a potent sign of patency; conversely, the near absence of distal run-off almost certainly indicated severe stenosis or total stent occlusion. The results of Cohen's K statistics shows that the interpretation of MSCT images for evaluating coronary stent patency has good inter-observer agreement.

We also showed that the intra-luminal CT attenuation value of restenotic stents was lower than that of non-restenotic stents. Furthermore, all the stents with relative value ([stent site]/[reference site]) less than 0.9 was revealed to have in-stent restenosis. Supporting our observation, Suzuki et al suggested an in vitro study that significant in-stent restenosis was indicated by lower luminal attenuation inside the stents than that in the unstented portion ${ }^{18} \mathrm{Al}-$ though it is impossible to precisely distinguish thrombus and intimal hyperplasia within the stent lumen in the present MSCT technology, we hope that as much as spatial and temporal resolution improves, it will become possible to quantitatively diagnose in-stent restenosis by using CT attenuation value.

Recently, drug-eluting stents (DES) were reported to be associated with a lower rate (3-5\%) of restenosis. ${ }^{19}$ In that regard, the very high specificity and negative predictive value for the detection of significant in-stent restenosis we obtained suggests that 16-slice MSCT could be highly useful for evaluating DES luminal patency. However, in routine clinical settings, many patients are treated with relatively small stents having diameters of $2.5 \mathrm{~mm}$ or $3.0 \mathrm{~mm}$. Further improvement in spatial resolution and temporal resolution will be necessary for MSCT to become a routine diagnostic procedure for the evaluation of stent patency (16-slice MSCT already has submillimeter spatial resolution, and the temporal resolution mainly depends on the gantry rotation time rather than on the algorithm used).
Limitations of MSCT for the assessment of stent patency include high heart rates, arrhythmias and disability to perform breath holding. Currently available 64-slice MSCT might overcome these technical limitations by improving temporal resolution as short as $160 \mathrm{~ms}$ and total scan time less than $5 \mathrm{~s}$.

\section{Conclusions}

The 16-slice MSCT can provide useful and valuable clinical information when evaluating stent patency during the chronic phase after implantation, especially in patients treated with a relatively large diameter coronary stent $(\geq 3.5 \mathrm{~mm})$. Further improvements in image quality will be necessary before MSCT is routinely used for follow up after coronary arterial stent implantation.

\section{References}

1. Antoniucci D, Valenti R, Santoro GM, Bolognese L, Trapani M, Cerisano $\mathrm{G}$, et al. Restenosis after coronary stenting in current clinical practice. Am Heart J 1998; 135: 510-518.

2. Mehran R, Dangas G, Abizaid AS, Mintz GS, Lansky AJ, Satler LF, et al. Angiographic patterns of in-stent restenosis: Classification and implications for long-term outcome. Circulation 1999; 100: $1872-$ 1878.

3. Maintz D, Juergens KU, Wichter T, Grude M, Heindel W, Fischbach R. Imaging of coronary artery stents using multislice computed tomography: In vitro evaluation. Eur Radiol 2003; 13: 830-835.

4. Schuijf JD, Bax JJ, Jukema JW, Lamb HJ, Warda HM, Vliegen HW, et al. Feasibility of assessment of coronary stent patency using 16slice computed tomography. Am J Cardiol 2004; 94: 427-430.

5. Serruys PW, de Jaegere P, Kiemeneij F, Macaya C, Rutsch W, Heyndrickx G, et al. A comparison of balloon-expandable-stent implantation with balloon angioplasty in patients with coronary artery disease: Benestent Study Group. N Engl J Med 1994; 331: 489-495.

6. Fischman DL, Leon MB, Baim DS, Schatz RA, Savage MP, Penn I, et al. A randomized comparison of coronary-stent placement and balloon angioplasty in the treatment of coronary artery disease: Stent Restenosis Study Investigators. N Engl J Med 1994; 331: 496-501.

7. Kimura T, Yokoi H, Nakagawa Y, Tamura T, Kaburagi S, Sawada $\mathrm{Y}$, et al. Three-year follow-up after implantation of metallic coronary-artery stents. $N$ Engl J Med 1996; 334: 561-566.

8. Kim WY, Danias PG, Stuber M, Flamm SD, Plein S, Nagel E, et al. Coronary magnetic resonance angiography for the detection of coronary stenoses. N Engl J Med 2001; 345: 1863-1869.

9. Pump H, Mohlenkamp S, Sehnert CA, Schimpf SS, Schmidt A, Erbel R, et al. Coronary arterial stent patency: Assessment with electron-beam CT. Radiology 2000; 214: 447-452.

10. Achenbach S, Giesler T, Ropers D, Ulzheimer S, Derlien H, Schulte $\mathrm{C}$, et al. Detection of coronary artery stenoses by contrast-enhanced, retrospectively electrocardiographically-gated, multislice spiral computed tomography. Circulation 2001; 103: 2535-2538.

11. Nieman K, Rensing BJ, van Geuns RJ, Munne A, Ligthart JM, Pattynama PM, et al. Usefulness of multislice computed tomography for detecting obstructive coronary artery disease. Am J Cardiol 2002; 89: $913-918$.

12. Sato $\mathrm{Y}$, Matsumoto N, Kato M, Inoue F, Horie T, Kusama J, et al. Noninvasive assessment of coronary artery disease by multislice spiral computed tomography using a new retrospectively ECG-gated image reconstruction technique. Circ J 2003; 67: 401-405.

13. Nieman K, Cademartiri F, Lemos PA, Raaijmakers R, Pattynama PM, de Feyter PJ. Reliable noninvasive coronary angiography with fast submillimeter multislice spiral computed tomography. Circulation 2002; 106: $2051-2054$.

14. Leclerc X, Gauvrit JY, Pruvo JP. Usefulness of CT angiography with volume rendering after carotid angioplasty and stenting. Am J Roentgenol 2000; 174: 820-822.

15. Becker CR, Ohnesorge BM, Schoepf UJ, Reiser MF. Current development of cardiac imaging with multidetector-row CT. Eur J Radiol 2000; 36: $97-103$

16. Nieman K, Oudkerk M, Rensing BJ, van Ooijen P, Munne A, van Geuns RJ, et al. Coronary angiography with multi-slice computed tomography. Lancet 2001; 357: 599-603.

17. Kuettner A, Trabold T, Schroeder S, Feyer A, Beck T, Brueckner A, et al. Noninvasive detection of coronary lesions using 16-slice multi- 
slice spiral computed tomography technology: Initial clinical results. J Am Coll Cardiol 20045; 44: 1230-1237.

18. Suzuki S, Furui S, Kaminaga T, Yamauchi T, Kuwahara S, Yokoyama N, et al. Evaluation of coronary stents in vitro with CT angiography: Effect of stent diameter, convolution kernel, and vessel orientation to the z-axis. Circ J 2005; 69: 1124-1131.
19. Morice MC, Serruys PW, Sousa JE, Fajadet J, Ban Hayashi E, Perin $\mathrm{M}$, et al. Randomized study with the sirolimus-coated by velocity balloon-expandable stent in the treatment of patients with de novo native coronary artery lesions: A randomized comparison of a sirolimuseluting stent with a standard stent for coronary revascularization. $N$ Engl J Med 2002; 346: 1773-1780. 